\title{
Caracterização elétrica de um Sensor de pH usando um Transistor de Efeito de Campo com Gate-Estendido.
}

\author{
William Max dos Santos Silva Silva ${ }^{1}$; Ernando Silva Ferreira ${ }^{2}$ \\ 1. Bolsista Probic/Uefs, Graduando em Bel.Física, Universidade Estadual de Feira de Santana, e-mail: \\ max.fisuefs@gmail.com \\ 2. Orientador, Departamento de Física, Universidade Estadual de Feira de Santana, e-mail: ernando@ yahoo.com
}

PALAVRAS-CHAVE: Egfet; Sensor; $\mathrm{pH}$.

\section{INTRODUÇÃO}

Devido as aplicações dos biossensores para o desenvolvimento dos mais variados segmentos como processamento de alimentos, farmácia, medicina entre outros, e com o avanço exponencial da microeletrônica, a cada dia esses sensores vêm se tornando mais baratos e compactos, possibilitando medidas confiáveis em tempo real tão seguras quanto as dos aparelhos empregados em laboratórios de análise. (Sugimoto L. 2003)

Sensores são dispositivos capazes de mensurar ou quantificar uma grandeza luminosidade, pressão, $\mathrm{pH}$ - a partir de um sinal físico ou químico. São compostos também por um transdutor, o qual converte um sinal de entrada para um domínio desejável, geralmente sinal elétrico (Balbinot, A.; Brusamarello.2011).

Os biossensores baseados na técnica de potenciometria proposta por Nernste em 1888 é uma das técnicas mais aplicadas para medidas de $\mathrm{pH}$, atividade fundamental para os mais diversos campos, uma vez que com este é possível fazer medidas indiretas de ureia, colesterol, glicose e etc. (Daniel R. Thévenot, at.al. 1999).

Com o advento dos transistores muitas pesquisas vêm surgindo a exemplo do Ion Sensitive Field Effect Transistor ISFET e suas aplicações como biossensor (Bergveld, P. 2003). Dentro desta perspectiva o trabalho propõe simular e caracterizar eletricamente uma membrana sensível a $\mathrm{H}^{+}$a partir de um transistor.

\subsection{O EgFet}

Em 1970 Bergveld (Bergveld, P. 1970, 2003) propõem pela primeira vez um sensor baseado em Fet, o Isfet, que é uma tecnologia cara e inflexível. (Silva, Gláucio Ribeiro. 2009)

Anos depois, um novo dispositivo similar ao ISFET foi criado, o Extended-gate field effect transistor (EGFET) (Van der Spiegel.1983). Este consiste basicamente um MOFET comercial com um elétrodo ligado ao gate, garantindo ao EGFET flexibilidade, uma vez que o elétrodo de trabalho pode ser substituído por qualquer outro, e os transistores usados são acessíveis e reutilizáveis, tornando-se um ótimo sensor de $\mathrm{pH}$.

Muitos testes são feitos para encontrar membranas que respondam a uma ampla faixa de $\mathrm{pH}$. Algumas características do material interferem na sensibilidade; uma delas é a cristalinidade. Nesse sentido, o Fluorine-doped Tin Oxide (FTO) apresenta particularidades interessantes (Diniz, Pablo .2008).

Os sensores de $\mathrm{pH}$ do tipo EGFET têm a sua corrente modulada a partir do campo elétrico gerado pelos íons $\left(\mathrm{H}^{+}\right)$da solução, os quais alteram o potencial entre a membrana e o eletrólito. Tendo como modelo para o sistema a teoria electrolyte insulator semiconductor 
(EIS), que leva em consideração a teoria Site-bending model e o funcionamento do ISFET, que também se aplica ao EGFET. Lembrando que o comportamento do Egfet só diverge do Mosfet na tensão de threshould $V_{T H}$ devido as contribuições do dipolo de superfície $\left(\chi_{s}\right)$, potencial de superfície $\left(\psi_{0}\right)$ devido a meio e potencial do eletrodo de referência $\left(E_{r e f}\right)$. Sendo assim, para o Mosfet na região não saturada (Resende, S. M. 1996)

$$
I_{D S}=\frac{\mu_{n} C_{o x} W}{L}\left[V_{G S}\left(V_{G S}-V_{T H}\right)-\frac{V_{D S}^{2}}{2}\right],
$$

Para região saturada

$$
I_{D S}=\frac{\mu_{n} C_{o x} W}{2 L}\left[\left(V_{G S}-V_{T H}\right)^{2}\right]
$$

Seja

$$
V_{T H}(\text { EGFET })=V_{T H}(\text { MOSFET })+E_{r e f}-\psi_{0}+\chi_{s}-\frac{\phi_{s}}{q} .
$$

$V_{F B}$ é a tensão de float band que depende da função trabalho do metal e do oxido, e $Q_{b}$ é a carga na região de depleção. No Egfet todo o sistema contribui para uma variação de $V_{T H}$.

\subsubsection{Site- bending model}

A forma com a qual o potencial do gate é modulada a partir da atividade de $\mathrm{H}^{+}$é explicada pelo modelo de sitio de ligações (site-bending model). Este proposto por Yets e generalizado por Fung em 1986. Neste modelo a membrana sensível, um óxido, têm sítios de ligações anfóteros e dada interação com o os prótons da solução formam-se sítios neutros, ácidos e básicos que podem ser descritos pelas constantes de equilíbrio ácido $\left(K_{a}\right)$ e base $\left(K_{b}\right)$.

podem ser escritos em função das características da superfície, $K_{a} K_{b}$ e número de sítios por área $N_{s}$. Seja o $p H_{p z c}$ ou $p H$ no ponto de carga zero, $\psi_{0}=0=\psi_{0}$.

$$
p H_{p z c}=-\log \left(K_{b} x K_{a}\right)^{\frac{1}{2}}=\frac{1}{2}\left(p K_{a}+p K_{b}\right),
$$

$\mathrm{O} \Psi_{0}$ é definido como:

$$
2,303\left(p H_{p z c}-p H\right)=\frac{q \Psi_{0}}{k T}+\sinh ^{-1}\left(\frac{q \Psi_{0}}{k T \beta}\right) ; \beta=\frac{2 q^{2} N_{s}\left(\frac{K_{a}}{K_{b}}\right)^{-1 / 2}}{C_{d l} k T} .
$$

Sendo $k$ a constande de Boltzman e, o parâmetro $\beta$ expressa a sensibilidade do óxido em função do $p H$, sobretudo a Eq.[4] é uma aproximação para superfícies nas quais o número de sítios é suficientemente grande assegurando que a densidade de carga não seja máxima na fixa de $p H$ a se trabalhar, para isso $\Delta p K=p K_{a}-p K_{b}$ é grande o suficiente para garantir que $\frac{K_{a}}{K_{b}} \ll 1$.

\section{METODOLOGIA}

Para caracterizar a membrana, no caso FTO, seguindo os procedimentos de literatura fora escolhido o CMOS (do inglês Complementary Metal Oxide Semiconductor) comercial, cd4007ube. Para caracterização do dispositivo fora selecionando um Fet tipo $\mathrm{N}$ que foi 
simulado no softwere livre LTSpice ${ }^{\circledR}{ }^{1}$. Tendo em mão fontes de 12 volts e divisores de tensão obtendo variação de 0,06 a 5 volts foi variando manualmente $V_{D S}$ a valores fixos de $V_{G S}$ e assim é possível observar variações de $I_{D S}$, deste modo plotar a curva característica $I_{D S}$ vs $V_{D S}$.

Com as relações entre $V_{G S}, \quad V_{D S}$ e $I_{D S}$ apresentadas nas Eq.[1,2] ,espera-se obter uma curva que se modifique de acordo com a tensão no gate, consequentemente com o pH. A partir destes dados e equações determinar a sensibilidade da membrana e comportamento em uma faixa de $p H$.

\section{RESULTADOS}

Tendo em vista a caracterização do transistor, a Figura $[3,4]$ apresentam simulações do dispositivo obtidas no softwere LTSpice®.

No gráfico da Figura[1] é possível visualizar o comportamento do transistor, ainda mais, nele é apresentado como a tensão de entrada controla a corrente de saída como também o $\mathrm{V}_{\mathrm{TH}}$. Para esta simulação é necessário fixar $\mathrm{V}_{\mathrm{DS}}$ e variar $\mathrm{V}_{\mathrm{GS}}$. A partir do esquema apresentado na Figura [1] variou-se $\mathrm{V}_{\mathrm{GS}}$ de 0 a 5 em passos de 0,01 Volts e $\mathrm{V}_{\mathrm{DS}}$ de 0 a 5 em passos de 1 Volts. A partir do esquema apresentado na Figura [1], variou-se $\mathrm{V}_{\mathrm{GS}}$ de 0 a 5, em passos de 0,01 Volts, e $V_{D S}$ de 0 a 5, em passos de 1 Volts.

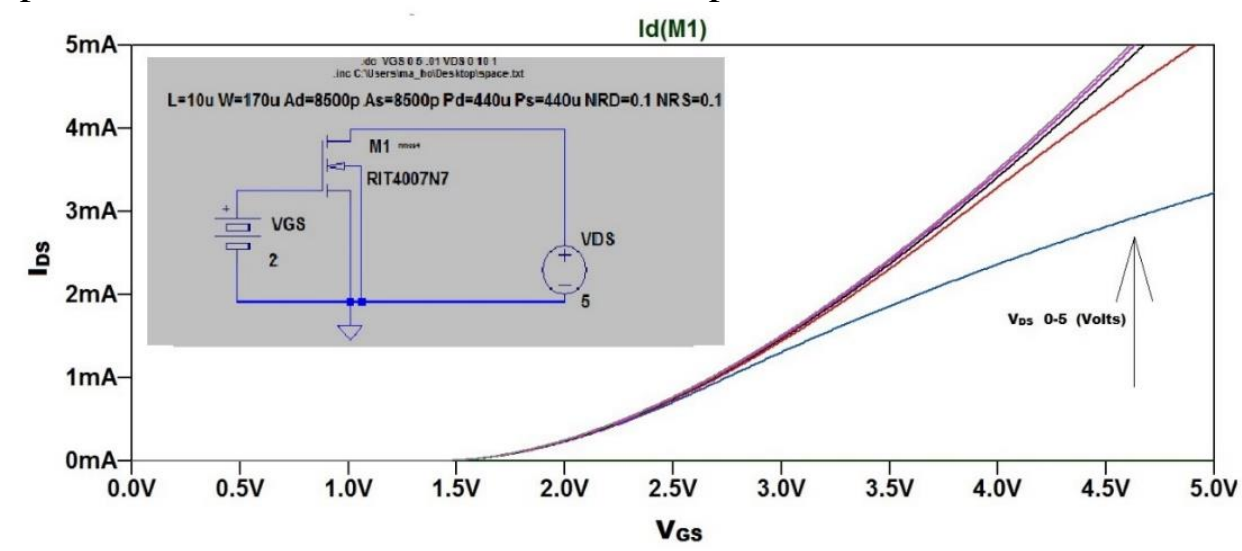

Figura 1. Simulação da curva de transcondutância do cd4007ube e esquema do circuito para obtenção dos dados.

Com o LTSpice e os parâmetros do cd4007 obtidos no Rochester Institute of Technology ${ }^{2}$ foi possível plotar a curva característica deste componente. Vide Figura [2]

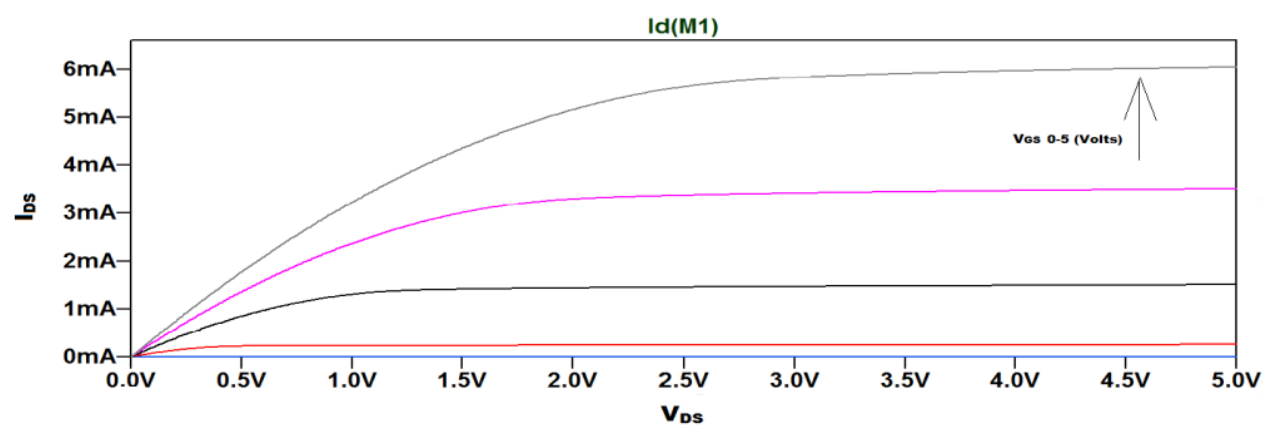

Figura 2 Simulação da curva característica do cd4007ube e esquema.

\footnotetext{
1 Disponível para download em <http://www.analog.com/en/design-center/design-tools-and calculators/ltspice-simulator.html $>$, acesso em 01/08/2018.

${ }^{2}$ Disponível em < https://people.rit.edu/lffeee/CD4007_SPICE_MODEL.pdf>, acesso em 01/08/2018.
} 
No gráfico apresentado na Figura [3] apresenta a curva características do transistor. Nesta é de fácil visualização das zonas: saturação, corte e tríodo. A ultima é uma zona de interesse neste trabalho. Tendo essas simulações como referência é possível uma melhor analise do gráfico apresentado na Figura [3].

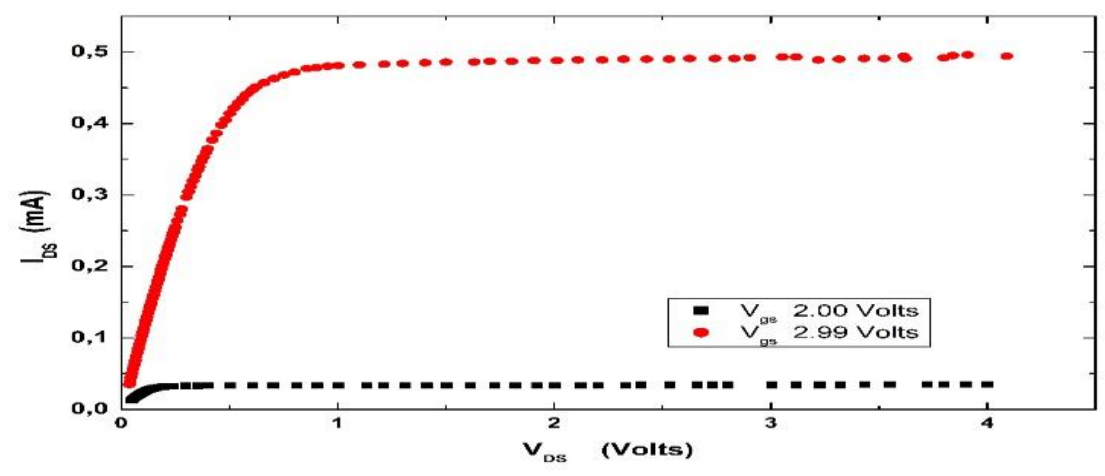

Figura 3 Curva característica experimental do cd4007ube.

Esta curva apresenta um resultado aceitável se observado seu comportamento. Deste modo o próximo passo é montar o EgFet e caracterizar a membrana, para assim calibrar o sistema possibilitando determinar a sensibilidade do eletrodo de trabalho. Isso não foi feito pois, ainda não se dispõem de um eletrodo de referência $\mathrm{Ag} / \mathrm{AgCl}$ no laboratório. Contudo, é possível obter uma relação linear entre o $\mathrm{pH}$ e a raiz quadrada de IDs, tendo em vista a Eq.[6] basta aplicar raiz quadrada em ambos os membros da equação. Assim se tem algo semelhantes a uma função do primeiro grau. Nesta garantia basta fixar um valor de $V_{D S}$ na zona de saturação e plotar uma curva $\sqrt{I_{D S}}$ vs $p H$.

Com a curva de transcondutância para valores fixos de $\mathrm{pH}$ é possível determinar a sensibilidade da membrana, para isso varia-se $V_{r e f}$ a $V_{D S}$, valores de $V_{D S}$ próximos ao $\mathrm{V}_{\mathrm{TH}}$, ou seja próximo o $\mathrm{pH}_{\mathrm{pzc}}$, constante é possível levantar uma curva $V_{\text {ref }} \mathrm{vs} \mathrm{pH}$, seja o valor de $I_{D S} \quad$ o limiar de condução. Nestas condições $\frac{q \Psi_{0}}{k T} \ll \beta$ observe que a Eq.[3] pode ser reescrita de tal forma que ao substituir Eq.[3] na Eq.[7] lembrando que $I_{D S}$ é constante

$$
C=\propto_{V_{D S}} V_{G S} ; \quad C=I_{D S}+S_{T, \beta}+S_{V_{T H}, V_{D S}}^{\prime}
$$

Deste modo o método se apresenta confiável e funcional, as dificuldades de materiais impediram a realização completa do experimento, sobretudo os estudos realizados mostram que é possível e existe uma robustez teórica.

\section{REFERÊNCIAS}

Bergveld, P.1970. Development of an Ion-Sensitive Solid-State Device for Neurophysiological Measurements. IEEE Transactions on Biomedical Engineering, Bm17,1,p.70-71

Van der spiegel, J., Lauks, I., Chan, P., Babic, D.1983. The extended gate chemically sensitive field effect transistor as multi-species microprobe. Sens. Actuators 4,p.291-298.

Daniel R. Thévenot,at.al.1999. Electrochemical Biosensors: Recommended Definitions and Classification ,Pure Appl. Chem., Vol. 71, No.12, p. 232-348.

Sugimoto L.2003.Biossensores:de tão simples e baratos, as pessoas desconfiam, Jornal da Unicamp, 232, p.8.

Bergveld, P. 2003. Thirty years of ISFETOLOGY: what happened in the past 30 years and what may happen in the next 30 years. Sens. Actuators B: Chem. 88 , p1-20.

Diniz, Pablo Batista.2008. Dispositivos semicondutores a partir de óxidos de estanho e zinco, Ribeirão Preto, pp. 171.

Silva, Gláucio Ribeiro.2009. Materiais micro e nanoestruturados para sensores de íons do tipo EGFET. Ribeirão Preto.p.6-33.

Balbinot, A.; Brusamarello, V.J.2011. Instrumentação e Fundamentos de Medidas 2a. ed. LTC,p.9-11. 